\title{
ADAM 12: A putative marker of oligodendrogliomas?
}

\author{
Dimitrios Kanakis $^{\mathrm{a}, *}$, Uwe Lendeckel ${ }^{\mathrm{e}}$, Paraskevi Theodosiou ${ }^{\mathrm{f}}$, Henrik Dobrowolny ${ }^{\mathrm{a}}$, \\ Christian Mawrin $^{\mathrm{b}}$, Gerburg Keilhoff ${ }^{\mathrm{d}}$, Alicia Bukowska ${ }^{\mathrm{c}}$, Knut Dietzmann ${ }^{\mathrm{b}}$, Bernhard Bogerts ${ }^{\mathrm{a}}$ and \\ Hans-Gert Bernstein ${ }^{\mathrm{a}}$ \\ ${ }^{a}$ Department of Psychiatry, Otto-von-Guericke-University, Magdeburg, Germany \\ ${ }^{\mathrm{b}}$ Istitute of Neuropathology, Otto-von-Guericke-University, Magdeburg, Germany \\ ${ }^{\mathrm{c}}$ Institute of Experimental Internal Medicine, Otto-von-Guericke-University, Magdeburg, Germany \\ ${ }^{\mathrm{d}}$ Institute of Medical Neurobiology, Otto-von-Guericke-University, Magdeburg, Germany \\ ${ }^{\mathrm{e}}$ Institute of Experimental Internal Medicine, Ernst-Moritz-Arndt-University, Greifswald, Germany \\ ${ }^{\mathrm{f}}$ Department of Pathology and Cytology, University Hospital of Larissa, Larissa, Greece
}

\begin{abstract}
ADAM 12 (meltrin alpha) belongs to a large family of molecules, consisting of members with both disintegrin and metalloproteinase properties. ADAMs have been implicated in several cell physiological processes including cell adhesion, cell fusion, proteolysis and signalling. ADAM 12 is widely expressed, including skeletal muscle, testis, bone, intestine, heart and kidney. In addition, a variety of tumours show elevated expression of ADAM12; among them being breast-, colon-, gastricand lung-carcinoma. As to the brain, ADAM 12 has been shown previously to be expressed in rat and human oligodendrocytes. However, little is known about the expression of this protease in brain tumours. This study demonstrates the presence of ADAM 12 in non-neoplastic oligodendroglial cells of normal human brain as well as in neoplastic oligodendroglia and minigemistocytes arising from four pure oligodendrogliomas and three mixed oligoastrocytomas. Double stainings revealed a notable preference of ADAM 12 for the oligodendroglial over astroglial components. The results of immunohistochemistry are in accordance with the results obtained from the RT-PCR, which further demonstrated a mild difference concerning the mRNA concentration of ADAM 12 between similar grades of eight astrocytomas and eight oligodendrogliomas (namely four astrocytomas grade II versus four oligodendrogliomas grade II and four astrocytomas grade III versus four oligodendrogliomas grade III). Both cellular immunostaining for ADAM 12 and ADAM 12 mRNA content decrease with higher histologic grade of the tumour. Surprisingly, the latter parameter (ADAM12 mRNA) showed a significant opposite correlation to the degree of histologic tumour malignancy. From our data showing that ADAM 12 is highly expressed in, but not restricted to, oligodendrogliomas, we conclude that ADAM 12 immunohistochemistry may be a helpful tool in the diagnosis of brain tumours.
\end{abstract}

Keywords: ADAM 12, gliomas, oligodendrogliomas, immunohistochemistry, RT-PCR

\section{Introduction}

ADAM (A Disintegrin And Metalloproteinase) proteins are known to be implicated in cell-cell and cell-

\footnotetext{
${ }^{*}$ Corresponding author: Dimitrios N. Kanakis, Specialist in Neuropathology and Resident in General Pathology, Democritus University of Thrace, Department of Pathology, University Hospital of Alexandroupolis, 68100 Alexandroupolis, Greece. Tel.: +30 6972425563; E-mail: aristoteles_stageira@yahoo.com.
}

matrix interactions, thus contributing to cell adhesion, cell migration, cell survival and proliferation $[11,16$, 27,35]. Since these features are essential prerequisites for cancer invasion and metastasis, a potential role of ADAMs in tumour development and expansion has been suggested. For instance, up-regulation of ADAM-9 has been associated with hepatocellular carcinoma [30], and the expression level of ADAM-9 mRNA and protein was significantly increased in both breast carcinomas and fibroadenomas [25]. ADAM12 
has been shown to be highly expressed in breast carcinoma cells [9] and to take part in the formation of multinucleated giant cell tumours of bone [31]. Gliomas comprise a large group of tumours with different cytomorphological characteristics, including astrocytomas, oligodendrogliomas, and ependymomas. Of particular importance for an accurate diagnosis and adequate treatment is the recognition of oligodendrocyte-derived neoplasms, since the clinical course and optimal therapeutic approach differs from that of other gliomas. Previous studies showed that oligodendrogliomas respond better to chemotherapy than astrocytomas of comparable grade [6,33]. Moreover, the presence of oligodendroglial features in high grade astrocytomas appears to be associated with a better prognosis $[5,10]$. Thus, the establishment of a reliable oligodendroglioma marker is of particular clinical importance.

However, a specific marker which specifically recognizes oligodendrogliomas only, but no other members of the glioma family, is still lacking. Among the proteins tested so far are cell type-specific marker proteins of mature oligodendroglial cells such as 2',3'cyclic nucleotide phosphodiesterase, myelin basic protein (MBP), myelin associated glycoprotein (MAG), myelin proteolipids, and galactolipids. Unfortunately, the expression patterns of these markers observed in oligodendroglial neoplasms considerably limit their application [12,22,29]. A second group of genes/proteins examined included those expressed in undifferentiated oligodendrocyte precursor cells such as chondroitin sulfate proteoglycan (NG2) and the alpha receptor of platelet-derived growth factor ( $\mathrm{PDGF} \alpha \mathrm{R})$ [4,7,17,23]. In a study performed by Shoshan et al. [28], both proteins were found to be differentially expressed in oligodendroglial tumours, but also in pilocytic astrocytomas and, though to a lesser extent, even in glioblastomas. Additionally, in vitro experiments have demonstrated that NG2 also detects so-called O-2A cells, which are generally accepted to be progenitors of both oligodendroglia and type 2 astrocytes. Under serum-free conditions, these precursor cells $(\mathrm{O}-2 \mathrm{~A})$ differentiate into oligodendroglial cell type, whereas in the presence of serum they develop to type 2 astrocytes [26].

Two independent reports proposed Olig 1 and Olig 2 to be useful oligodendroglia markers that showed only faint expression in other gliomas [19,20]. However, recent studies demonstrated similar protein levels of both Olig 1 and 2 in oligodendrogliomas and astrocytomas [3,24].

We have demonstrated that ADAM 12 is specifically localized in rat and human oligodendrocytes [2], and in this context we thought that this protein could be used as a possible and exclusive marker in the recognition of oligodendrogliomas. However, a relatively recent report demonstrating overexpression of ADAM 12 also in human glioblastomas [14], seems to argue against a selective role of this protein in the formation and progression only of oligodendrogliomas. Hence, the present study was undertaken to learn more about the occurrence of this metalloprotease in various human glial neoplasms. Special attention was paid to oligodendroglial cell-derived tumours.

\section{Materials and methods}

\subsection{Normal human brain tissue}

All brains were obtained from pathologists or medical examiners with full consent of each family and in accordance to the ethics and rules outlined by German law and the local ethics committee of the University of Magdeburg. Brains of four individuals without neurological or psychiatric disorders (two males, 49 and 63 years, two females, 54 and 61 years) were studied. Brains were removed within 48 hours after death. The tissue preparation was as described in another relevant study, which was carried out by our group [2]. Briefly, brains were fixed in toto in $8 \%$ phosphate-buffered formaldehyde ( $\mathrm{pH}$ 7.0). After embedding the brains in Paraplast, serial coronal sections were cut on a microtome and mounted. Every $50^{\text {th }}$ section was stained for morphological orientation (Nissl and HeidenhainWoelcke). Sections containing the prefrontal and the orbitofrontal cortex as well as subcortical white matter were studied.

\subsection{Tumour tissues and glial cell lines}

Thirty two formalin-fixed, paraffin-embedded biopsy samples were used for immunohistochemical investigations. All samples were retrieved from the files of the Institute of Neuropathology, University of Magdeburg. Specimens were accumulated between 1996 and 2003. Diagnoses were established according to the 2007 World Health Organization (WHO) classification of brain tumours using conventional H\&E stained sections, together with appropriate immunohistochemical markers [13]. For sixteen cases, additional frozen tumour material which had been snap-frozen in liquid nitrogen and stored at $-80^{\circ} \mathrm{C}$ was available. We studied by immunohistochemistry five diffuse astrocytomas 
(WHO grade II), six anaplastic astrocytomas (WHO grade III), five glioblastomas multiforme (WHO grade IV) without an apparent oligodendroglial component in H\&E stained sections, five oligodendrogliomas (WHO grade II), six anaplastic oligogendrogliomas (WHO grade III), and five oligoastrocytomas (WHO grade II).

The human malignant glioma cell line U138MG was purchased from the American Type Culture Collection (ATCC) and grown to $80 \%$ confluency in Earl's modified Eagle's medium (EMEM) from PAA laboratories (Linz, Austria), supplemented with 10\% heatinactivated foetal calf serum (FCS, PAA).

\subsection{Immunohistochemistry}

For immunohistochemistry, $4-\mu \mathrm{m}$-thick paraffin sections of the tumour specimens were dewaxed with xylene for $15 \mathrm{~min}$ and dehydrated through a series of graded alcohols. Sections were pretreated in a microwave oven using $0.01 \mathrm{M}$ sodium acetate buffer ( $\mathrm{pH}$ 6.0) for 3 $\times 10 \mathrm{~min}$. Endogenous peroxidase activity was blocked by incubation ( $30 \mathrm{~min}$ ) in $0.3 \% \mathrm{H}_{2} \mathrm{O}_{2}$ in methanol. The sections were gently rinsed with TBS-buffer and then incubated with bovine serum albumin for 30 minutes to reduce non-specific binding. Sections were incubated with a mouse monoclonal antibody against human ADAM 12 (BIOMOL, dilution: 1:400). Negative controls included omission of the primary antibody, its substitution by an irrelevant mouse monoclonal antibody and preabsorption of the antiserum by the synthetic peptide which was used to generate the antibody (peptide SA-378 from Biomol, for details see [2]). The signal was detected using the streptavidin-biotin-peroxidase complex method according to the manufacturer's recommendation (DAKO, Hamburg, Germany). DAB (3, 3 'diaminobenzidine hydrochloride containing $0.08 \%$ hydrogen peroxide) was used as a chromogen to visualize the peroxidase activity. Finally, the sections were counterstained with hematoxylin-eosin. In case of human brain sections the colour reaction was enhanced by ammonium sulfate solution as described in our previous study [2]. Glial fibrillary acidic protein (GFAP) was immunolocalized on consecutive sections to those immunostained for ADAM 12 by using a monoclonal antibody (DAKO). The primary dilution was 1:100 in phosphate buffered saline (PBS). The visualization of the reaction product was as for ADAM12. For three selected cases out of five of oligoastrocytomas double immunostainings for ADAM12 and GFAP were carried out using alternative staining protocols and chromogens (Ventana AEC [3-amino-9-ethylcarbazole] Basic Detection-Kit from Vetor Labs. yielding intense red-purblish colour, combined with 3, 3'-diaminobenzidine yielding brown colour). The infrequent occurrence of pale yellowishgreen metachromasia of AEC pointing to lower concentration of the protein to be detected was considered as recommended by Koretz et al. [15].

For confocal imaging of ADAM 12 localization in the malignant human glioma cell line U138MG, cells were grown for 24 hours on poly-L-lysine-treated microscopic slides. The cells were fixed in ice-cold $4 \%$ paraformaldehyde (PFA), followed by staining for ADAM 12 and glial fibrillary acidic protein (GFAP; DAKO, Hamburg; 1:100). Cy3 and Red-x (both at 1:50 dilution; Jackson ImmunoResearch, West Grove, PA) were used as secondary antibodies. Slides were then mounted in DPX (Fluka, Neu-Ulm, Germany), coverslipped, and examined using a Leica TCS-NT laser scanning confocal microscope (Heidelberg, Germany).

\subsection{Semi-quantitative evaluation of ADAM 12 and GFAP immunostainings in tissues}

The density of ADAM 12 immunoreactive oligodendrocytes in normal human brain tissue was estimated by counting stained cell profiles using a counting grid. The percentage of ADAM 12 expressing oligodendroglial cells was calculated by comparing the number of immunostained cells with the total number of oligodendroglial cells after counterstaining with Nissl [2]. Immunolabeling of other cell types was not observed. Due to the quite uneven distribution of ADAM12 and GFAP immunoreactive cells within individual tumours, the use of a counting grid was not ingenious. Instead, two independent investigators blinded to the diagnosis (D. K. and H.-G. B.) carefully inspected each staining pattern and assessed the number of immunoreactive objects regardless of intensity of intracellular staining. [between (0): no immunostained and (++++): $>75 \%$ immunostained neoplastic cells]. By comparing the ratings thereafter an inter-rater reliability (the relative observed agreement between raters) of about 0.9 was achieved.

\subsection{Real-time PCR}

Total RNA was extracted from a total number of sixteen frozen samples, eight oligodendrogliomas (grades II and III, four samples for each grade) and eight astrocytomas (grades II and III, four samples for each grade) using the RNeasy Mini Kit from Qiagen (Hilden, Germany) following the recommended protocol. The re- 
Table 1

ADAM 12 immunoexpression in human gliomas. The semi-quantitatively assessed percentage of immunostained neoplastic cells is given with crosses in brackets

\begin{tabular}{lcc}
\hline Diagnosis (N) & ADAM12 Immunostaining & GFAP Immunostaining \\
\hline Oligodendroglioma WHO grade II (5) & $5 / 5(+++)$ & $2 / 5(+)$ \\
Anapl. Oligodendrogl. WHO grade III (6) & $6 / 6(++)$ & $5 / 6(+)$ \\
Oligoastrocytoma WHO grade II (5) & $5 / 5(++)$ & $5 / 5(++)$ \\
Diffuse astrocytoma WHO grade II (5) & $3 / 5(2: 0 ; 1:++; 2:+)$ & $5 / 5(+++)$ \\
Anapl. astrocytoma WHO grade III (6) & $3 / 6(3: 0 ; 3:+)$ & $6 / 6(+++)$ \\
Gliobl. multiforme WHO grade IV (5) & $3 / 5(2: 0 ; 2:+; 1:++)$ & $5 / 5(+++)$ \\
\hline
\end{tabular}

N number of cases; Percentage of immunostained neoplastic cells $(0):$ no positive cells, $(+):<25 \%,(++): 25-50 \%,(+++): 51-75 \%,(++++)$ : $>75 \%$. Immunostaining expression in more than $75 \%$ of neoplastic cells was not observed in any of the tumours studied.

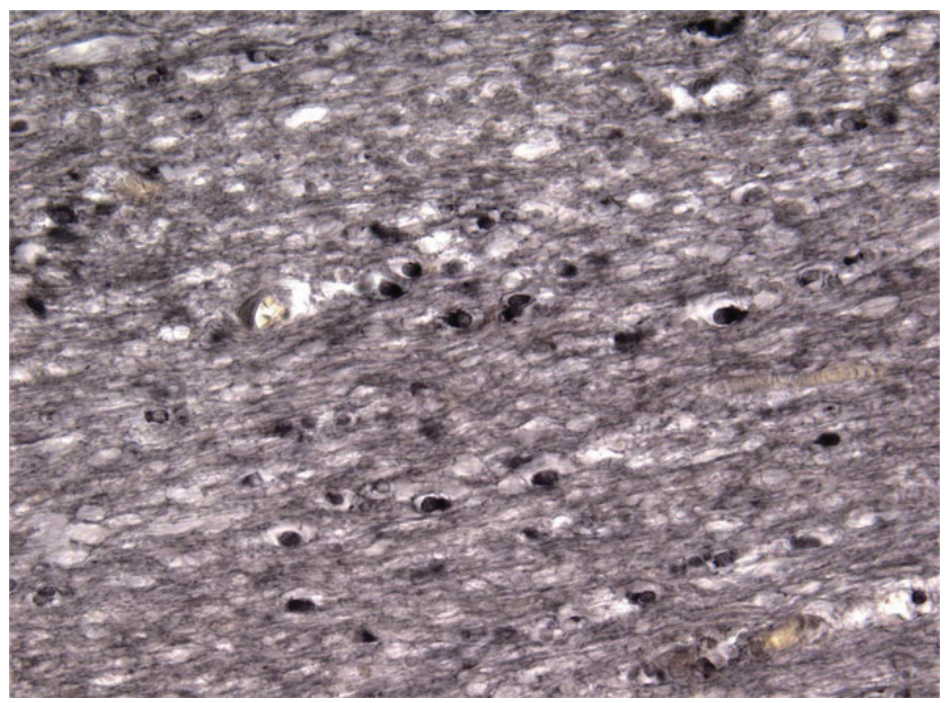

Fig. 1. ADAM 12 immunoreactive white matter oligodendrocytes in normal human postmortem brain. (Magnification $\mathrm{x} 400)$. (Colours are visible in the online version of the article; http://dx.doi.org/10.3233/DMA-120953)

sulting RNA was quantified spectrophotometrically using a GeneQuant (Pharmacia LKB, Freiburg, Germany) and stored at $-80^{\circ} \mathrm{C}$ until further required. One $\mu \mathrm{g}$ total RNA was transcribed into first strand cDNA using AMV reverse transcriptase (Promega, Mannheim, Germany) and random hexanucleotides (Roche Diagnostics, Germany), according to Arndt et al. [1].

Quantitative PCR was performed on one twentieth of the reverse transcription reaction, using the iCycler (real-time PCR device, Bio-Rad, Munich, Germany). All samples were analyzed in triplicate. A $25 \mu \mathrm{l}$ reaction mixture consisted of $12.5 \mu \mathrm{l}$ of HotStarTaq MasterMix (Qiagen, Hilden, Germany), $0.25 \mu$ l of Fluorescein Calibration Dye (BioRad, Munich, Germany), $0.25 \mu \mathrm{l}$ of SYBR Green I (Molecular Probes, Eugene, Oregon) and $0.5 \mu \mathrm{mol} / \mathrm{L}$ of the specific primers for ADAM 12 (forward: 5'- gCTgATgAAgTTgTCAgTgC; reverse: 5'-gAgACTgACTgCTgAATCAg). Primers were synthesized by BioTeZ (Berlin, Germany). Initial denaturation and activation of Taq polymerase at $95^{\circ} \mathrm{C}$ for
15 min was followed by 40 cycles with denaturation at $94^{\circ} \mathrm{C}$ for $30 \mathrm{sec}$, annealing at $62^{\circ} \mathrm{C}$ for $30 \mathrm{sec}$, and elongation at $72^{\circ} \mathrm{C}$ for $30 \mathrm{sec}$. A final extension step was performed at $72^{\circ} \mathrm{C}$ for $5 \mathrm{~min}$. An aliquot $(7 \mu \mathrm{l})$ of each reaction mixture was applied to a $1.4 \%$ agarose gel, electrophoresed in Tris-acetate buffer and then stained with ethidium bromide. ${ }^{\circledR}$-actin mRNA amounts were used to normalize cDNA contents for any variability in RNA amounts or integrity (forward: 5'- AAgATgACCCAgATCATgTTTgAg; reverse: 5'AggAggAgCAATgATCTTgATCTT).

\section{Results}

On normal human brain sections we were able to confirm our previous result that about $75 \%$ of all gray matter oligodendrocytes and about $60 \%$ of white matter oligodendrocytes express immunohistochemically detectable ADAM 12 (Fig. 1) [2]. 


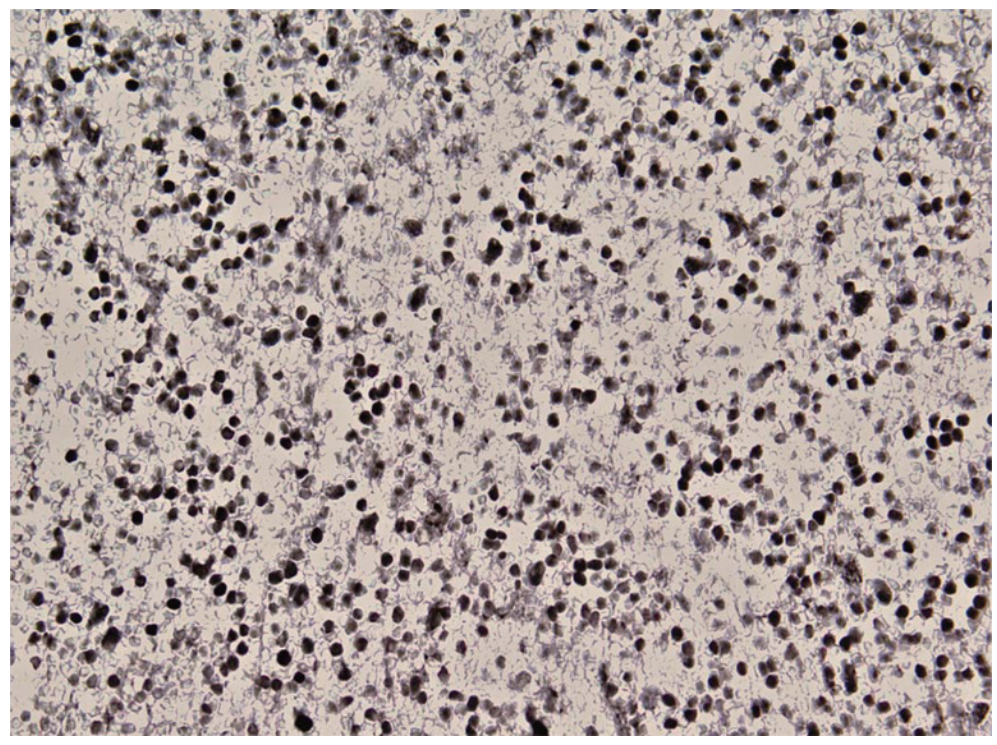

Fig. 2. Oligodendroglioma grade II. A vast majority of cells expresses ADAM 12. (Magnification x200). (Colours are visible in the online version of the article; http://dx.doi.org/10.3233/DMA-120953)

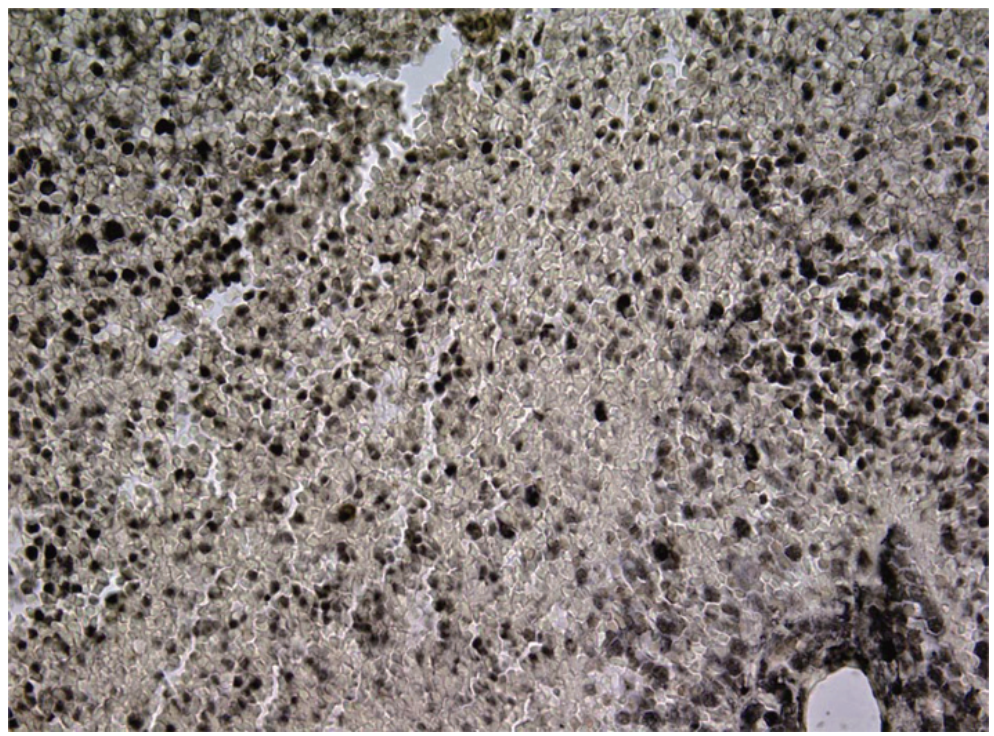

Fig. 3. Oligodendroglioma grade III. Many cells are immunoreactive for ADAM 12. (Magnification x200). (Colours are visible in the online version of the article; http://dx.doi.org/10.3233/DMA-120953)

The distribution of immunohistochemical ADAM 12-expression in common astrocytic and oligodendroglial tumours is summarized in Table 1. The immunohistochemical analysis revealed the presence of ADAM 12 in both low grade (WHO Grade II) and anaplastic (WHO Grade III) oligodendrogliomas and, though to a lesser extent, also in the diffuse (WHO Grade II) and anaplastic (WHO Grade III) astrocytomas which showed the well-known distribution pattern of
GFAP. With regard to oligodendrogliomas, ADAM 12 was seen in all cases under investigation. However, the immunohistochemical expression pattern of the enzyme protein was dependent on the grade of the tumour on one hand, and on the topography of the tumour on the other. Invasion margins of the tumors stood out by a more intense staining. The number of cells that stained positive for ADAM 12 in the grade II oligodendrogliomas approximately equaled that in 


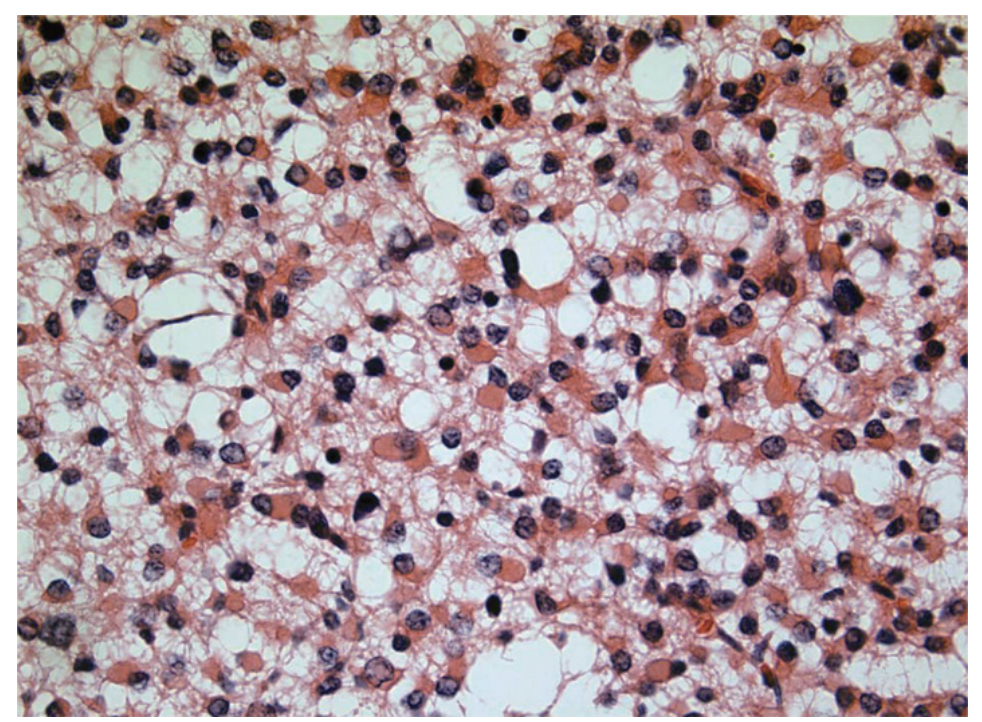

Fig. 4. A great number of "minigemistocytes", with their characteristic histologic features, regionally localized within an oligoastrocytoma $(H \& E$; Magnification $\times 400$ ). (Colours are visible in the online version of the article; http://dx.doi.org/10.3233/DMA-120953)

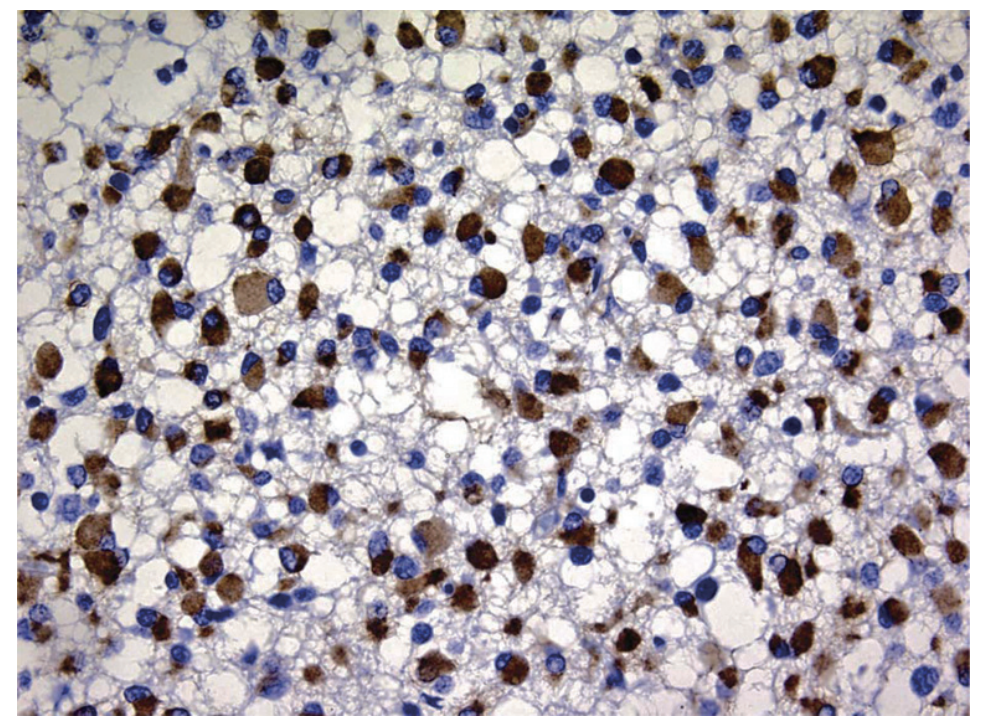

Fig. 5. The majority of "minigemistocytes" in Fig. 4 showed strong positivity for GFAP (Magnification x400). (Colours are visible in the online version of the article; http://dx.doi.org/10.3233/DMA-120953)

non-neoplastic brain tissue; about $70 \%$ of cells strongly expressed the protein (Fig. 2). In grade III oligodendrogliomas, a fraction of about $40-50 \%$ of all oligodendrocytes were found to contain ADAM12 immunoreactive material (Fig. 3).

In case of oligoastrocytomas (also called mixed gliomas), the antibody for ADAM 12 bound specifically to a subpopulation of neoplastic cells, which were predominantly localized in such areas corresponding to a characteristic histological oligodendroglial pat- tern (so called "honeycomb" or "chickenwire" appearance). In one case diffuse positive ADAM staining was found within a region consisting almost entirely of "minigemistocytes" with round to oval, eccentrically situated nuclei and abundant globoid cytoplasm lacking cell processes (Fig. 4). These cells were in their majority positive for GFAP (Fig. 5).

Interestingly, in these mixed tumours no co-localization of ADAM 12 and GFAP was observed (i.e. these two antibodies bound to different cells, thus recogniz- 


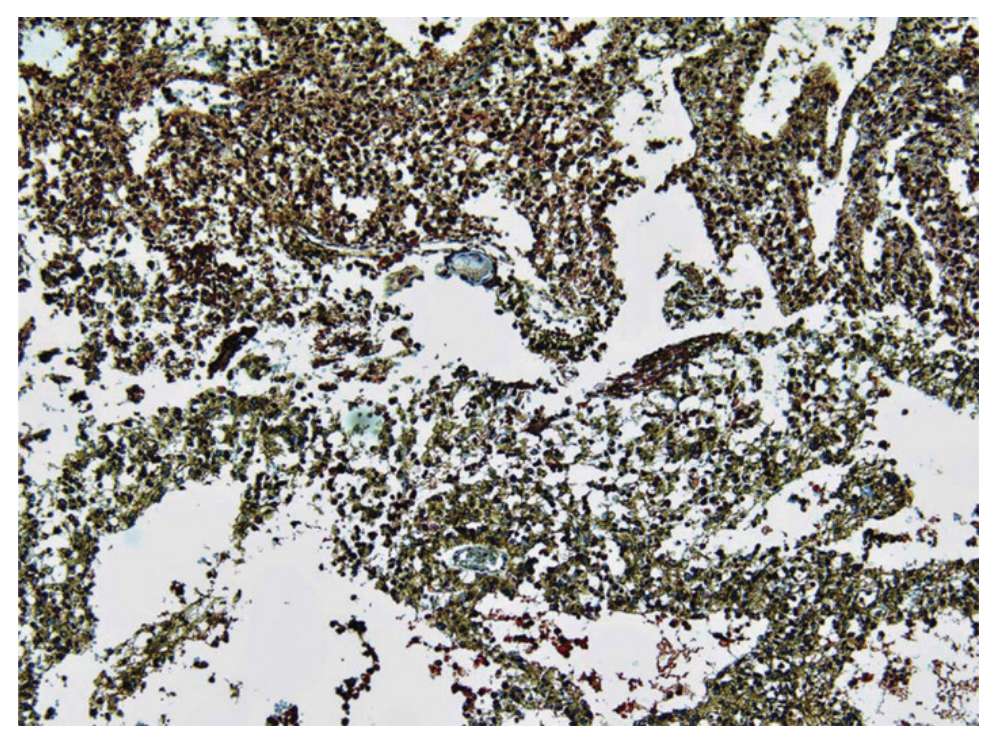

Fig. 6. A case of an oligoastrocytoma with an apparent border between the two consisting entities, demonstrated here as obviously separated staining patterns of ADAM12 and GFAP. Double immunostaining with antibodies against ADAM 12 (red colour) and GFAP (brownish colour) proteins. (Magnification x100). (Colours are visible in the online version of the article; http://dx.doi.org/10.3233/DMA-120953)

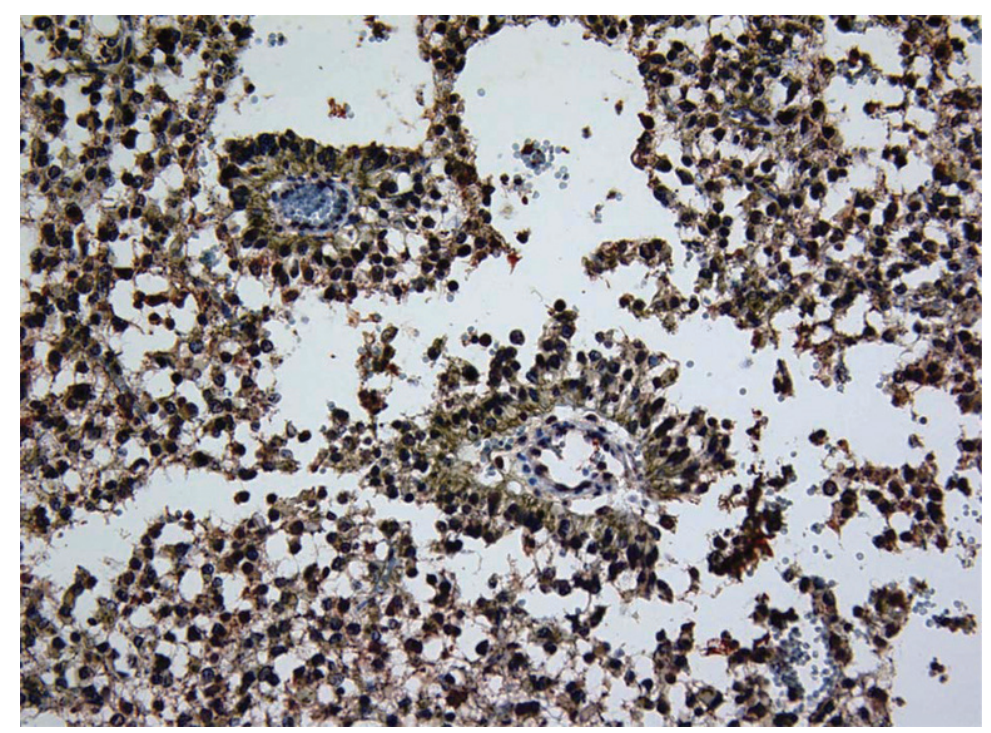

Fig. 7. GFAP positive astrocytes (brownish colour) with a prominent and characteristic perivascular localization, whereas in the surrounding neoplastic tissue mixed populations of GFAP positive astrocytes and ADAM12 positive oligodendroglia are diffusely intermingled. (Magnification $\mathrm{x} 200$ ). (Colours are visible in the online version of the article; http://dx.doi.org/10.3233/DMA-120953)

ing distinct tumour entities). The ability of ADAM 12 and GFAP antisera to discriminate different cell types is demonstrated within areas of no obvious architectural characteristics of one or the other tumour cell type. On the contrary, in regions where both tumour entities (astrocytic and oligodendroglial) are almost entirely separated from each other on grounds of typical histological patterns, the expression of ADAM 12 and GFAP demonstrated clear borders (Fig. 6). Double staining for ADAM12 and GFAP revealed that GFAP neoplastic cells were prominently localized around vessels (Fig. 7). In fact, anti-GFAP stains cells that possess the typical characteristics of normal astrocytes or their neoplastic counterparts, whereas anti-ADAM 12 recognises another population of cells which in turn does not express GFAP. However, in case of oligoastro- 


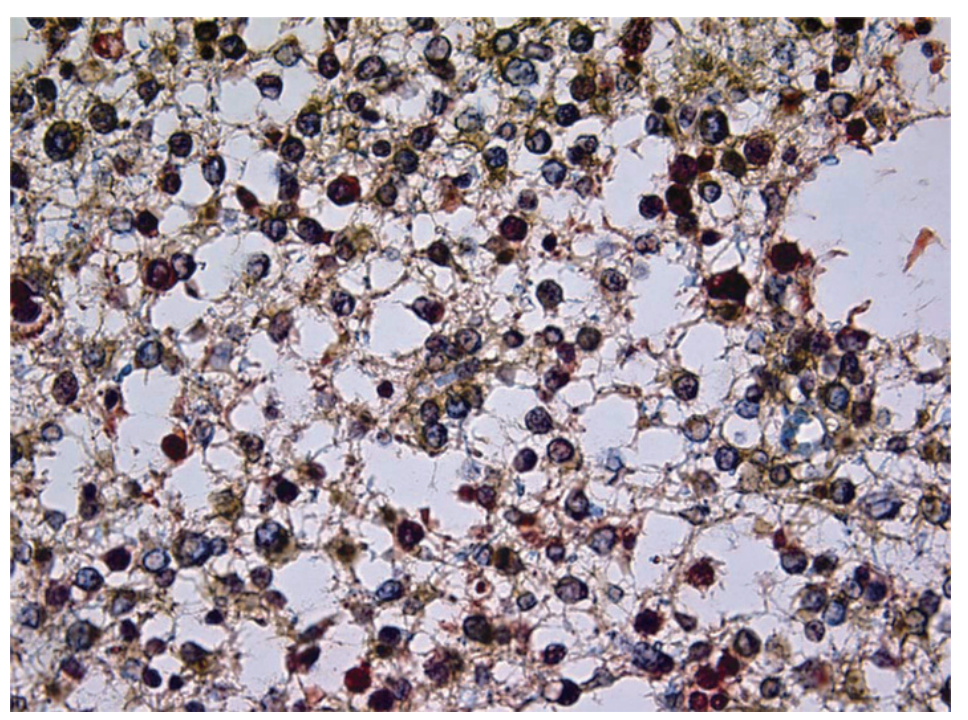

Fig. 8. Double immunostaining (ADAM 12 and GFAP) in an oligoastrocytoma showing an area with the typical "honeycomb" ("chickenwire") structure. Note that different cell populations are labelled. (Magnification x400). (Colours are visible in the online version of the article; http://dx.doi.org/10.3233/DMA-120953)

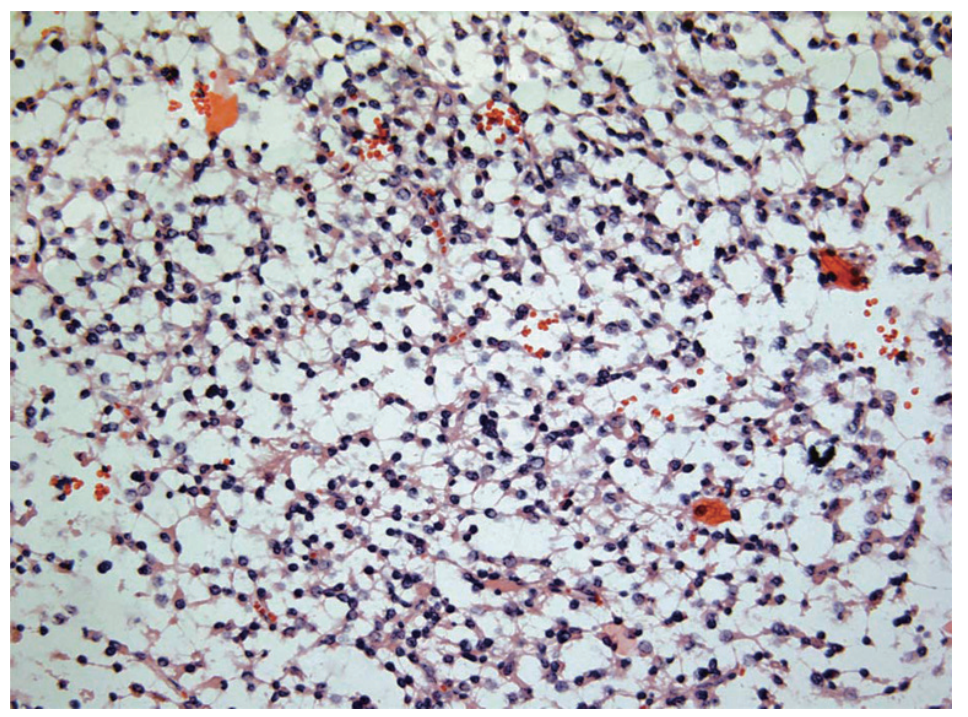

Fig. 9. Another case of an oligoastrocytoma with a "honeycomb" ("chickenwire") pattern, which according to Fig. 8 consists of two immunohistochemically distinct subpopulations of oligodendroglia cells, which however show no obvious morphological differences in routine H\&E staining (H\&E; Magnification x200). (Colours are visible in the online version of the article; http://dx.doi.org/10.3233/DMA-120953)

cytomas, it was obvious that within areas of the tumour where the typical "honeycomb" ("chickenwire") pattern of oligodendrogliomas could be seen, there were also some cells which stained for GFAP next to the ADAM 12 positive cells (Fig. 8). These cells had the same morphological characteristics as the ADAM 12 positive oligodendroglia, namely, round, centrally located nuclei surrounded by scanty perinuclear cytoplasm without prominent cell processes. It could be speculated that these cells belong to a subpopulation of oligodendroglia which are morphologically indistinguishable from their "close neighbours" in the routine H\&E preparations (Fig. 9), from which they differ only in the immunostaining profile, namely expression of GFAP instead of ADAM 12.

In some tumours ADAM 12 immunoreactivity was also associated with blood lymphocytes which is in accordance with observations made by us and others $[2$, 


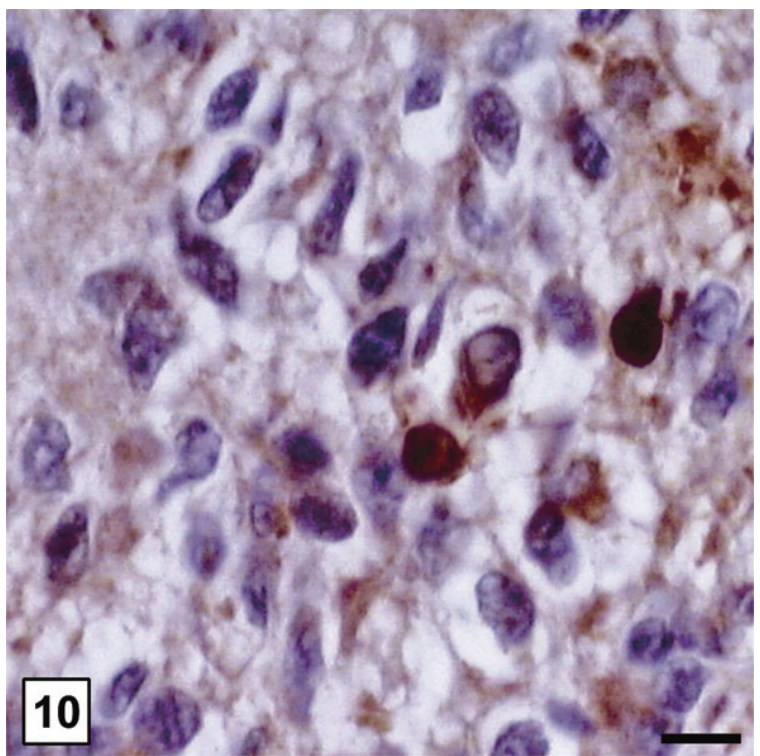

Fig. 10. Presence of ADAM 12 immunoreactive cells in a glioblastoma. $\mathrm{Bar}=15 \mu \mathrm{m}$. (Colours are visible in the online version of the article; http://dx.doi.org/10.3233/DMA-120953)

32]. ADAM 12-immunoreactive tumour cells were also seen in three of five glioblastomas (Fig. 10). In cultured U138MG glioma cells both ADAM 12 and GFAP were found to be equally well expressed both in percentage of stained cells and intensity of immunostaining. Moreover, the two stains showed a noticeable overlapping (Fig. 11 A-D).

Quantitative RT-PCR revealed the presence of only mild differences in the amounts of ADAM 12 mRNA in oligodendrogliomas and astrocytomas when analyzed independently of grading. However, by comparing low grade with high grade tumours, about 18 fold higher ADAM 12 mRNA amounts were detected in the low grade samples $(2917 \pm 1191$ vs. $157 \pm 133$ units, $P<$ 0.05) (Fig. 12).

\section{Discussion}

The present study describes for the first time the consistent expression of ADAM 12 in oligodendroglial tumours irrespective of their histological grading. Our preliminary results demonstrate a fairly specific recognition of neoplastic oligodendrocytes both in pure oligodendrogliomas and in oligoastrocytomas (mixed gliomas), whereby the antiserum apparently detects a subpopulation of neoplastic oligodendrocytes rather than the entire tumour specimen. This was evident in pure oligodendrogliomas as well as in oligoastro-

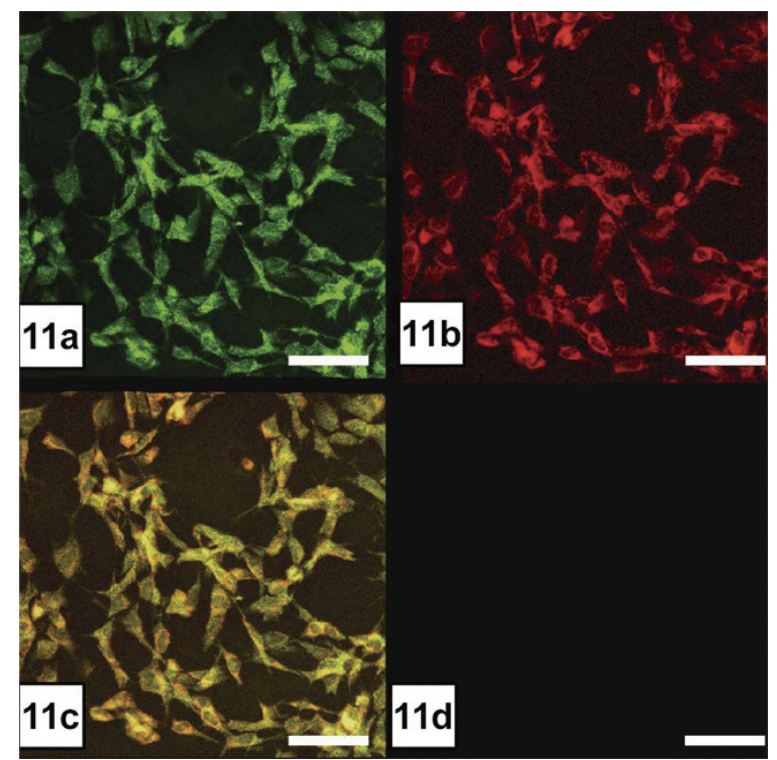

Fig. 11. Glioma cell culture. Bar $=60 \mu \mathrm{m}$. 11a. ADAM 12; 11b. GFAP; 11c. Merged; 11d. Control (omission of both antisera). (Colours are visible in the online version of the article; http://dx.doi. org/10.3233/DMA-120953)

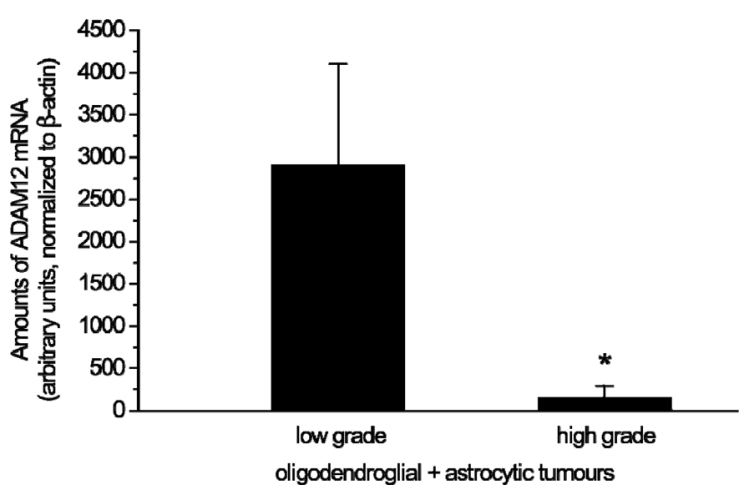

Fig. 12. ADAM 12 mRNA-expression of low and high grade oligodendroglial and astrocytic tumours. Low grade tumours contain about 18 fold higher ADAM mRNA amounts than their high grade counterparts $\left({ }^{*} P=0.04, n=7\right)$.

cytomas. The latter tumours showed an obvious difference between two otherwise cytomorphologically undistinguishable oligodendroglia subpopulations that are intermingled with each other in the typical oligodendroglial areas. One of them showed exclusively positive staining for ADAM 12, whereas the other appeared entirely positive for GFAP. There was no overlap between these two oligodendroglial cell groups since a co-localization of the two antibodies was not observed. The presence of GFAP positive cells within neoplastic oligodendrocytes has to be regarded regular and has been quite extensively documented in previous re- 
ports by others $[8,21,34]$. In a study presented by Herpers and Budka [8] the term "gliofibrillary oligodendrocytes" (gfoc) was used exclusively to describe these cells and the term gliofibrillary oligodendroglioma was subsequently introduced for the neoplasms consisting largely of gfocs. These so called "transitional" cells, which include the gliofibrillary oligodendrocytes and the minigemistocytes, were encountered to a variable extent and number either separately or in combination in all oligodendrogliomas, and only in one oligoastrocytoma. Consequently, this oligoastrocytoma should be named "transitional oligoastrocytoma" to better distinguish it from the classical oligoastrocytomas that are composed mainly of two distinct and non-transitional cell populations.

Both immunohistochemistry and quantitative RTPCR revealed highest expression levels of ADAM 12 in low grade tumours. These findings are at variance to results which were recently reported by Kodama et al. [14] in that these authors saw a selective overexpression of ADAM 12 in human glioblastoma compared to non-neoplastic brain tissues, low grade and anaplastic astrocytic tumours as well as intracranial neurinomas. It must be emphasized, however, that the mentioned investigators did not include oligoastrocytomas into their study. Interestingly enough, this group did not detect significant amounts of ADAM 12 in non-neoplastic human brain material. Our immunohistochemical and molecular biological investigations ([2] and the present study) show that ADAM 12 is consistently expressed in most oligodendrocytes of the normal brain, although its precise function remains to be elucidated fully. Our data regarding the evolution from lower to higher grade also suggest a specific role of ADAM 12 within a narrow range of early tumour development. The possible contribution of ADAM 12 in specific tumour functions such as growth, invasion, and angiogenesis should be addressed in future studies.

In summary, we propose that ADAM 12 may be helpful in identifying and quantifying large subpopulations of oligodendroglial cells in oligodendrogliaderived tumours and in mixed tumours. Its specificity and selectivity is quite comparable to that of other previously established markers of oligodendroglioma, such as Olig 1 and Olig $2[18,20,24]$. Hence, the search for a really selective immunohistochemical marker for oligodendrogliomas must be continued.

\section{Acknowledgments}

This research was supported by NBL-3 of BMBF of Germany and Stanley-Foundation.
We thank Mrs. S. Funke and Mr. M. Fanariotis for skilful technical assistance.

\section{References}

[1] M. Arndt, U. Lendeckel, C. Röcken, K. Nepple, C. Wolke, A. Spiess, C. Huth, S. Ansorge, H.U. Klein, A. Goette, Altered Expression of ADAMs (A Disintegrin And Metalloproteinase) in Fibrillating Human Atria, Circulation 105 (2002), 720-725.

[2] H.-G. Bernstein, G. Keilhoff, A. Bukowska, A. Ziegeler, S. Funke, H. Dobrowolny, D. Kanakis, B. Bogerts, U. Lendeckel, ADAM (A Disintegrin and Metalloprotease) 12 is expressed in rat and human brain and localized to oligodendrocytes, $J$ Neurosci Res 75 (2004), 353-360.

[3] C. Bouvier, C. Bartoli, L. Aguirre-Cruz, I. Virard, C. Colin, C. Fernandez, J. Gouvernet, D. Figarella-Branger, Shared oligodendrocyte lineage gene expression in gliomas and oligodendrocyte progenitor cells, J Neurosurg 99 (2003), 344-350.

[4] F. Di Rocco, R.S. Carroll, J. Zhang, P.M. Black, Plateletderived growth factor and its receptor expression in human oligodendrogliomas, Neurosurgery 42 (1998), 341-346.

[5] B. Donahue, C.B. Scott, J.S. Nelson, M. Rotman, K.J. Murray, D.F. Nelson, F.L. Banker, J.D. Earle, J.A. Fischbach, S.O. Asbell, L.E. Gaspar, A.M. Markoe, W. Curran, Influence of an oligodendroglial component on the survival of patients with anaplastic astrocytomas: a report of Radiation Therapy Oncology Group 83-02, Int J Radiat Oncol Biol Phys 38 (1997), 911-914.

[6] D. Fortin, G.J. Cairncross, R.R. Hammond, Oligodendroglioma: an appraisal of recent data pertaining to diagnosis and treatment, Neurosurgery 45 (1999), 1279-1291.

[7] I.K. Hart, W.D. Richardson, C.H. Heldin, B. Westermark, M.C. Raff, PDGF receptors on cells of the oligodendrocytetype-2 astrocyte (O-2A) cell lineage, Development 105 (1989), 595-603.

[8] M.J. Herpers, H. Budka, Glial fibrillary acidic protein (GFAP) in oligodendroglial tumors: gliofibrillary oligodendroglioma and transitional oligoastrocytoma as subtypes of oligodendroglioma, Acta Neuropathol 64 (1984), 265-272.

[9] K. Iba, R. Albrechtsen, B.J. Gilpin, F. Loechel, U.M. Wewer, Cysteine-rich domain of human ADAM 12 (meltrin alpha) supports tumor cell adhesion, Am J Pathol 154 (1999), 14891501.

[10] Y. Ino, M.C. Zlatescu, H. Sasaki, D.R. Macdonald, A.O. Stemmer-Rachamimov, S. Jhung, D.A. Ramsay, A. von Deimling, D.N. Louis, J.G. Cairncross, Long survival and therapeutic responses in patients with histologically disparate highgrade gliomas demonstrating chromosome 1p loss, J Neurosurg 92 (2000), 983-990.

[11] P. Keely, L. Parise, R. Juliano, Integrins and GTPases in tumour cell growth, motility and invasion, Trends Cell Biol $\mathbf{8}$ (1998), 101-106.

[12] P.G. Kennedy, B.A. Watkins, D.G. Thomas, M.D. Noble, Antigenic expression by cells derived from human gliomas does not correlate with morphological classification, Neuropathol Appl Neurobiol 13 (1987), 327-347.

[13] D. Louis, H. Ohgaki, O. Wiestler W. Cavenee Eds. World Health Organization Classification of Tumours of the Central Nervous System. International Agency for Research on Cancer (IARC) Press Lyon, 2007.

[14] T. Kodama, E. Ikeda, A. Okada, T. Ohtsuka, M. Shimoda, T. Shiomi, K. Yoshida, M. Nakada, E. Ohuchi, Y. Oka- 
da, ADAM12 is selectively overexpressed in human glioblastomas and is associated with glioblastoma cell proliferation and shedding of heparin-binding epidermal growth factor, $A m$ J Pathol 165 (2004), 1743-1753.

[15] K. Koretz, J. Leman, I. Brandt, P. Möller, Metachromasia of 3-amino-9-ethylcarbazole (AEC) and its prevention in immunoperoxidase techniques, Histochemistry 86 (1987), 471478.

[16] D.A. Lauffenburger, A.F. Horwitz, Cell migration: A physically integrated molecular process, Cell 84 (1996), 359-369.

[17] J.M. Levine and A. Nishiyama, The NG2 chondroitin sulfate proteoglycan: A multifunctional proteoglycan associated with immature cells, Perspect Dev Neurobiol 3 (1996), 245-259.

[18] K.L. Ligon, J.A. Alberta, A.T. Kho, J. Weiss, M.R. Kwaan, C.L. Nutt, D.N. Louin, C.D. Stiles, D.H. Roswitch, The oligodendroglial lineage marker OLIG2 is universally expressed in diffuse gliomas, J Neuropathol Exp Neurol 63 (2004), 499509.

[19] Q.R. Lu, J.K. Park, E. Noll, J.A. Chan, J. Alberta, D. Yuk, M.G. Alzamora, D.N. Louis, C.D. Stiles, D.H. Rowitch, P.M. Black, Oligodendrocyte lineage genes (OLIG) as molecular markers for human glial brain tumors, Proc Natl Acad Sci USA 98 (2001), 10851-10856.

[20] Y. Marie, M. Sanson, K. Mokhtari, P. Leuraud, M. Kujas, JY. Delattre, J. Poirier, B. Zalc, K. Hoang-Xuan, OLIG2 as a specific marker of oligodendroglial tumour cells, Lancet 358 (2001), 298-300.

[21] A.C.O. Meneses, J.J. Kepes, N.H. Sternberger, Astrocytic differentiation of neoplastic oligodendrocytes, J Neuropathol Exp Neurol Suppl 41 (1982), S 368.

[22] Y. Nakagawa, E. Perentes, L.J. Rubinstein, Immunohistochemical characterization of oligodendrogliomas: An analysis of multiple markers, Acta Neuropathol 72 (1986), 15-22.

[23] A. Nishiyama, X.H. Lin, N. Giese, C.H. Heldin, W.B. Stallcup, Co-localization of NG2 proteoglycan and PDGF alphareceptor on $\mathrm{O} 2 \mathrm{~A}$ progenitor cells in the developing rat brain, J Neurosci Res 43 (1996), 299-314.

[24] A. Ohnishi, H. Sawa, M. Tsuda, Y. Sawamura, T. Itoh, Y. Iwasaki, K. Nagashima, Expression of the oligodendroglial lineage-associated markers Olig1 and Olig2 in different types of human gliomas, J Neuropathol Exp Neurol 62 (2003), 10521059 .

25] C. O'Shea, N. McKie, Y. Buggy, C. Duggan, A.D. Hill, E. McDermott, N. O'Higgins, M.J. Duffy, Expression of ADAM9 mRNA and protein in human breast cancer, Int J Cancer 105 (2003), 754-761.

[26] M.C. Raff, R.H. Miller, M. Noble, A glial progenitor cell that develops in vitro into an astrocyte or an oligodendrocyte depending on culture medium, Nature 303 (1983), 390-396.

[27] C.D. Roskelley, A. Srebrow, M.J. Bissell, A hierarchy of ECM-mediated signalling regulates tissue-specific gene expression, Curr Opin Cell Biol 7 (1995), 736-747.

[28] Y. Shoshan, A. Nishiyama, A. Chang, S. Mörk, G.H. Barnett, J.K. Cowell, B.D. Trapp, S.M. Staugaitis, Expression of oligodendrocyte progenitor cell antigens by gliomas: Implications for the histogenesis of brain tumors, Proc Natl Acad Sci USA 96 (1999), 10361-10366.

[29] J. Szymas and A. Wajgt, Myelin-associated glycoprotein (MAG) in oligodendrogliomas. An immunohistochemical study, Neuropatol Pol 23 (1985), 239-246.

[30] A. Tannapfel, K. Anhalt, P. Häusermann, F. Sommerer, M. Benicke, D. Uhlmann, H. Witzigmann, J. Hauss, C. Wittekind, Identification of novel proteins associated with hepatocellular carcinomas using protein microarrays, J Pathol 201 (2003), 238-249.

[31] B.L. Tian, J.M. Wen, M. Zhang, D. Xie, R.B. Xu, C.J. Luo, The expression of ADAM12 (meltrin alpha) in human giant cell tumours of bone, Mol Pathol 55 (2002), 394-397.

[32] H. Toft-Hansen, R.K. Nuttall, D.R. Edwards, T. Owens, Key metalloproteinases are expressed by specific cell types in experimental autoimmune encephalomyelitis, J Immunol $\mathbf{1 7 3}$ (2004), 5209-5218.

[33] M.J. van den Bent, New perspectives for the diagnosis and treatment of oligodendroglioma, Expert Rev Anticancer Ther 1 (2001), 348-356

[34] J.D. van der Meulen, H.J. Houthoff, E.J. Ebels, Glial fibrillary acidic protein in human gliomas, Neuropathol Appl Neurobiol 4 (1978), 177-190.

[35] K.M. Yamada, B. Geiger, Molecular interactions in cell adhesion complexes, Curr Opin Cell Biol 9 (1997), 76-85. 


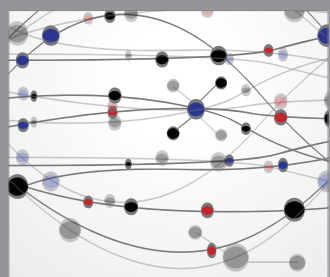

The Scientific World Journal
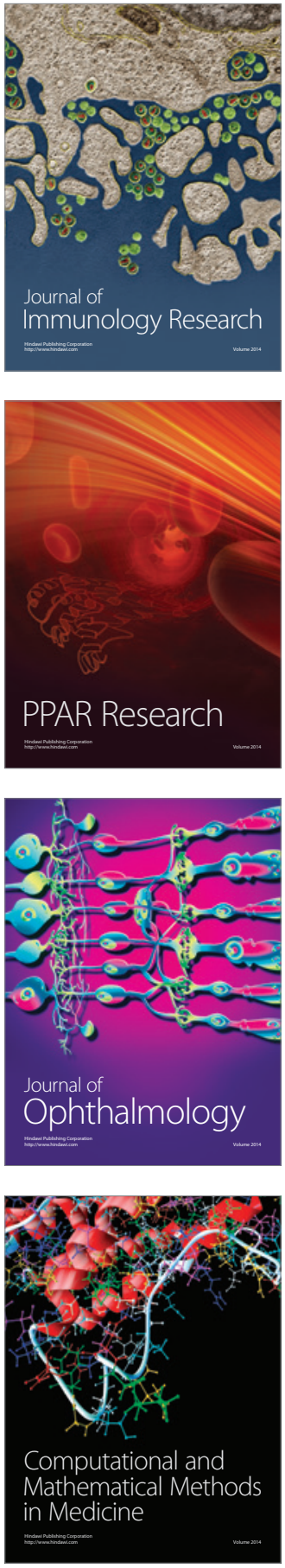

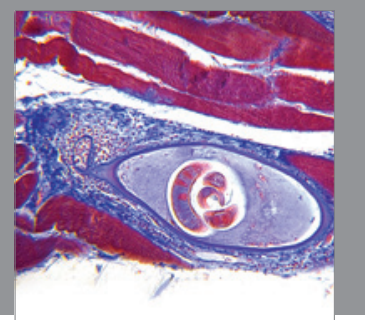

Gastroenterology

Research and Practice
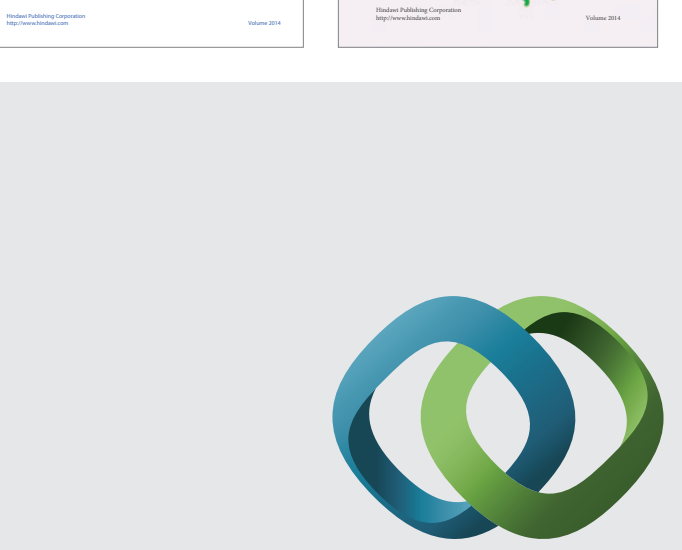

\section{Hindawi}

Submit your manuscripts at

http://www.hindawi.com
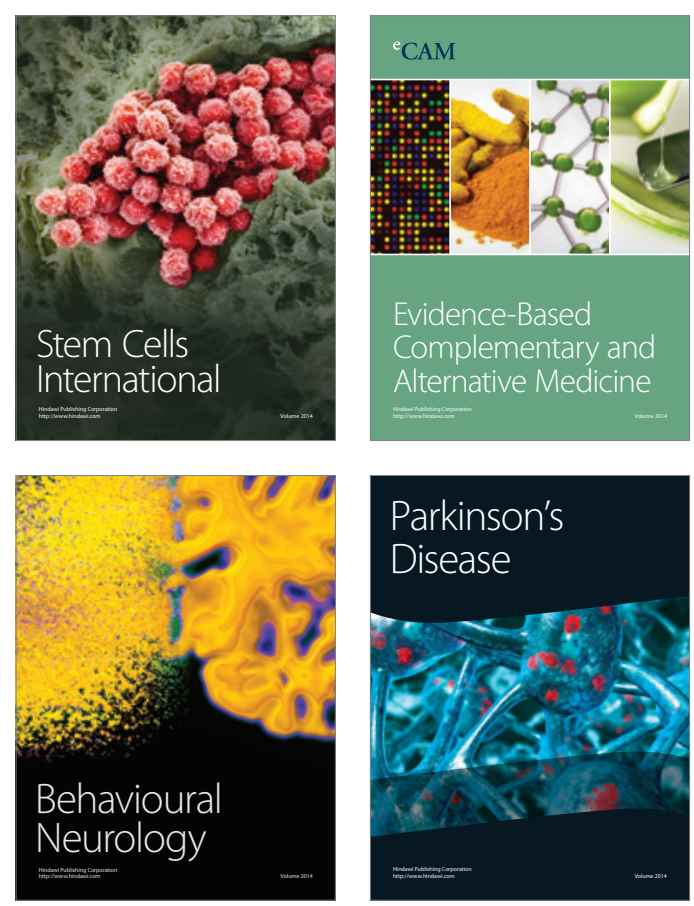

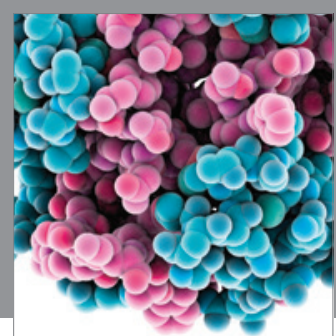

Journal of
Diabetes Research

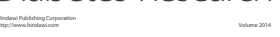

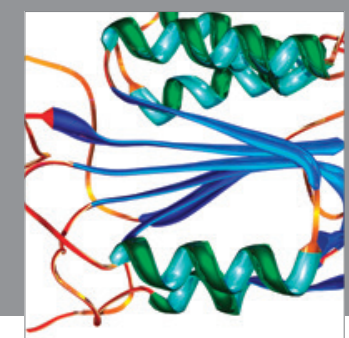

Disease Markers
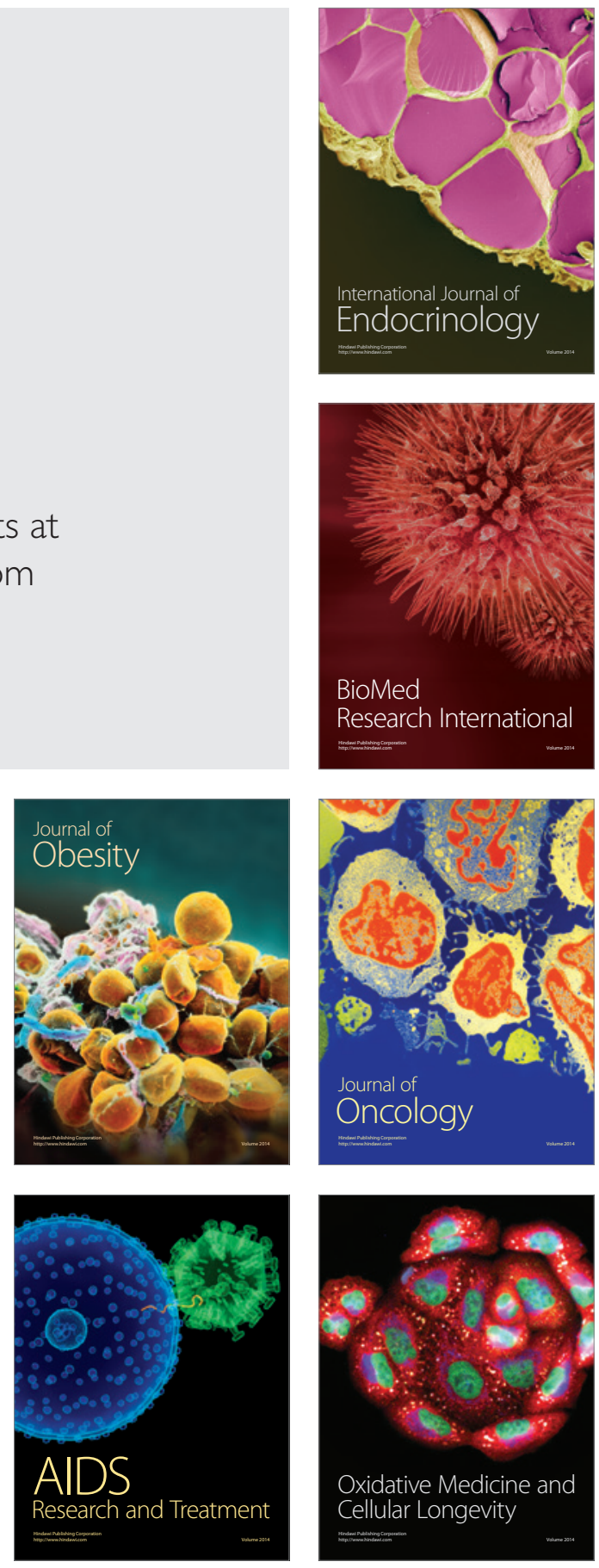